\title{
Design of Bus Station Based on Sustainable Development and Humanization Designprinciples-Take Two Design Plans for Example
}

\author{
Jianhua Lyu, Jia Xiang, Junqi Zhao and Ming \\ College of Forestry, Sichuan Agricultural University, Chengdu, Sichuan,611130, China \\ Email: ljh@sicau.edu.cn
}

\begin{abstract}
This study was performed to investigate the status and exiting problems of bus station design use by using comparative analysis, literature analysis, case analysis and other methods, then provide references for functional requirements of bus station. The theoretical and practical analysis says that principle of sustainability, demand, safety, humanization and cultural heritage should be considered in bus station design. Finally, the research results of this study were demonstrated by the case of two bus station design plans, and the development trend of bus station design are forecasted in order to provide certain scientific basis for the design of bus station in the future.
\end{abstract}

\section{Introduction (Heading 1)}

With the development of China's economy, people's living standards continue to rise, and their demands are changing. Heavy traffic, air pollution and other issues become increasingly serious, government strengthens environmental protection and puts forward the strategy of sustainable development in order to prevent the pollution of the environment.[1].

In the construction of modern cities, the construction of public facilities should meet the coordinated development of the economy, society, environment and culture in four aspects. As an important component of urban public facilities, bus station takes on many social functions, such as conveying public awareness, displaying economic culture and embodying aesthetic consciousness[2]. With the continuous development of society and the rapid growth of floating population, urban public transport has become a means of transportation for non-native population, and the bus station has become an important platform for publicizing the image of the city[3]. It has a direct impact on the psychology and behavior of the general public. However, there are still many problems in the design of the bus station at this stage, which cannot meet the needs of people. With the changing needs of citizens, more and more people are eager to live with the public facilities that are richer in human nature, whichplays a role in relieving physical and mental exhaustion and relaxing yourself [4].

Therefore, on the basis of the government's sustainable development strategy, it is an urgent problem for the bus station to grasp the humanization designconcept[5], and constantly optimize its own value and meet the needs of the contemporary people.

\section{Design status of bus station at present stage}

Public Transport Stations, that is bus station, is the product of urban development, the corresponding design proposals are drawn upbased on the status of the city frequently, and unified with urban planning.

Although the bus stations can satisfy the basic function of the urban environment at the present stage, but it lacks the cultural connotation and individual character of the city as a whole. The appearance of bus station looks all in the same key, which not only do not play the role of beautifying the city, but also leavepassengers cannot feel the feeling of freshness[6].Solving the general decorative structural problems by space modeling and color matching simply leads no breakthrough innovation, which ignores discussion of cultural heritage and integrity[2]. As a whole, the spiritual connotation of the bus station is not given, and the cultural and artistic characteristics of the bus station cannot be taken into account.

In addition, urban residents have weak awareness of the public transport station, and their participation in design opinions is low[7], resulting in the bus station is still unable to fully meet the wishes of people, the damages of stations are serious, design strategy for fast lane that leads tothe frequency of safety accidents increases. At this stage, although the bus station can ensure the realization of basic functions, but new technologies and concepts are still lacking in development [8]. 


\section{Problems existing in the design of bus station}

Lack of security considerations. At this stage, the bus station meet their basic functions at the same time without considering the parking area. When the bus pulled into the station, passengers are crowded with each other in order to get on the bus as soon as possible, sometimes passengers could be pushed down the station, if bus has no time to brakepersonal injuries could be caused.There is no obvious boundaries between bus station and pavement, and the bus station is easily to get destroyed.

Ignore special group. Bus station not only need to consider the general passenger situation, but also should consider special groupsituation, such as children who are not tall enough, old people who are not easy to move and parents who are carrying their children, which are not fully considered in current bus station design status[8]. There are some shortages like lighting intensity of the stop sign is not high, no English version in the stop sign, no access for physically handicapped, which could not meet the needs of special groups.

Lack of humanity. The bus station failed to satisfy the humanization design completely, such as text is too small in the stop sign to recognize, no public seat and ceiling are designedas rest and shade parts for passengers, large advertising area resulting in the bus route map occupied and the size of the bus station is not considered according to the flow of people.

Lack of sustainable development policy.The ecological environment is facing great challenges, in recent years, Chinese government promotes vigorously the idea of building modern ecological civilized society based on the concept of sustainable development and ecological urban.The use of new energy sources, such as solar energy, wind energy and biomass energy, has not yet been fully popularized in bus station design. Single function, high manufacturing cost, unreasonable use of materials and low recycling rate lead to the failure to achieve sustainable development in a comprehensive way. With the progress and development of society, people's demand for the bus station constantly changing, the design requirements of the bus station are also rising.

Under the environment of sustainable development, the design concept can only follow the principle of sustainable development. On this basis of innovation and development, so that the bus station can better meet the needs of modern people.

Large touch screen games are designed intobus stations inSan Francisco, which reduce the boredom of waiting for bus by incorporating interesting and intelligent design [14]. The bus station near the Vitoria insect paradise simulated the insect's visual system, which allow passengers to intuitively see their image in the insect's eyes [1]. This kind of bus station is loved by people, so we can see that the combination of form and function to meet people's needs is the trend of the future bus station design.
In recent years, traffic accident occurred frequentlyaround the bus station, which mainly due to few consideration for the parking area in bus station design, The passengers see the bus approaching in the distance, in order to get on the bus as soon as possible, has crowded to the road, sometimes passengers could be pushed down the station, if bus has no time to brakepersonal injuries could be caused. Safety is also an important principle to avoid the occurrence of safety accidents.

The bus stationdesign is an integrated complex, therefore, the local design should be unified with the whole. Not only the local style should be unified with the whole city, but also capacity design requires the area vehicle flowrate and visitors flowrate as the baseline, so as to effectively promote the relationship between people and people, structure and function.The theoretical and practical analysis says that (1) raise the ground height of the bus station to form an independent space to facilitate people to get on and off, (2) the ground is paved with non slip material to prevent the station from slipping in rainy days and (3) take full account of regional characteristics to determine roof height and extension width in order to achieve better realization of its sunshade, shelter function.

In the aspect of bus station design, we need carry on a systemic comprehension to our traditional culture and find the new consensus and valuable inheritance. The bus station in the town of Cornwall, so-called one of most beautiful bus stations in Britain, changes decorations and chairs according to different themes each year, which meet the needs of the people desire. Culture is a common symbol of a country or region. The development of the country cannot be separated from the promotion of culture. As an important component of urban construction, the bus station bears the important task of carrying forward the culture. Therefore, the future bus station will take the urban culture as the trend.

\section{Analysis and demonstration of design principles of bus station}

The design of bus station needs to be realized their own value as much as possible from peopleorientedperspective with the introduction of the integration of contemporary advanced technology and principle of sustainable development.

From the point of view of sustainable development, combined with the basic principles and methods for design of bus station, the two plans of bus stations are designedas design demonstration to find out the development trend of the future bus station.

The earth is a complex giant system, and each country and region is an integral subsystem of this huge system[10]. Any local error will directly affect the earth as a whole, in order to maintain their own relatively stable development and growth, common development, coordinated development and equitable development are put forward to ensure the sustainable development, including energy and resources. 

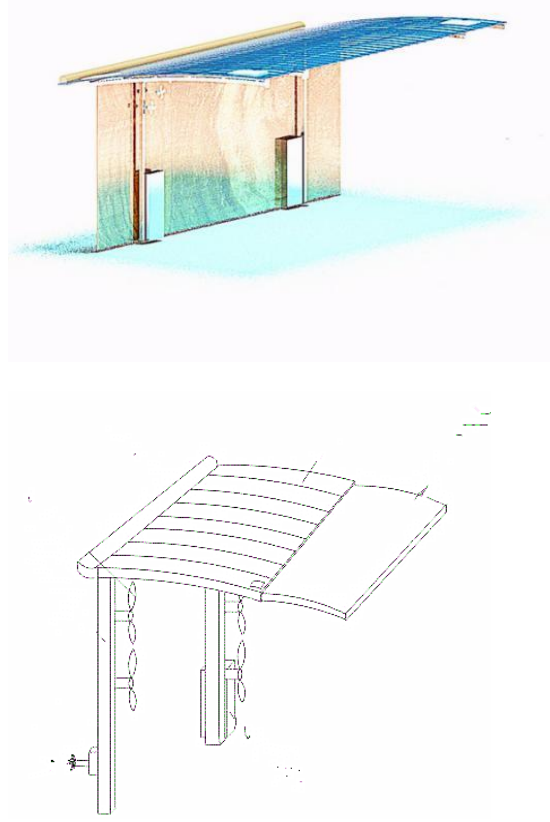

Figure 1. Design plan number one of bus station.

As shown in Figure 1, the design demonstration are set up tomeet the conditions of sustainable development. solar panels are designed on the roof of bus station, which will absorb solar energy and turn it into electrical energy by the storage device. The top sun visor will be stretched automatically with turning on induction switchaccording to the sunlight shining area by incorporating the sun induction device, which could protect the passengers from the sun. In addition, the rainwater collection device and impact force transmission system could make the rotor in the magnetic induction devicerotation with fallingrain, which would cause coils produce current and store that in batteries for sun visor expansion, fan rotation and rainwater treatment equipment using. The collected rainwater could form water vapor through rainwater treatment apparatus to cool down the surrounding environment of the bus station. When water vapor could not be used to cooling environment, the fans could run to accelerate the circulation of air around the bus station and hot air would be dispersed to achieve rapid cooling purposes, which reduce heat stroke, physical discomfort and other risks [12].

This design plan is aimed at the heat caused by outdoor weather, incorporate rainwater collection and treatment equipment into the ceiling by turning falling rainwaterimpact into electricity, at the same time, rainwater is collected and purified so as to achieve a physical cooling effect. The overall design produceno energy losses, energy conservation and protection to meet sustainable development by using a long-term and recyclable manner.

The humanization design principle has certain development prospects and trends in design field, which is a reflection on science and technology. Instead of emphasizing material, efficiency and technology, we should start with the characteristics of human psychology and physiology, making people in harmony with the environment [9].

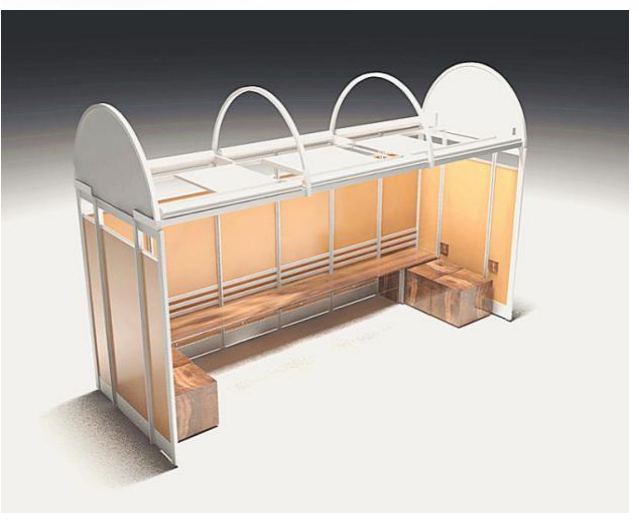

Figure 2. Design plan number two of bus station

As shown in Figure 2, the design demonstration are set up tomeet the conditions of humanization design. Incorporated into the design are the temperature converter, solar energy absorption device, rainwater collection device, impurity induction device and electrical storage device. Solar energy is collected and converted into electrical energy stored in a storage bottle for charging for passengers.

Temperature converters are designed on the roof of bus station, temperature from the roof under the sunshine heats the collected rainwater into warm water, then warm water flows into the evaporating box, the warm water is changed into flowing steam in a low pressure environment by regulating the pressure, which promote the turbine rotation and start AC motor power generation and storage into battery. The converted steam regulates the temperature around the platform. When impurities designed on the roof of bus station are induced to more than $90 \%$, cleaning device is activated automatically. Telescopic stools are designed at the two ends of bus station to provide passengers with rest facilities [13].

This plan mainly aims at combating boredom when waiting for the bus and the bus platform easy to dirty, cleaning device, charging plug and telescopic stool are designed into bus station, which make sure people charge their phones and rest in station. The whole design is based on humanization design and integrated into the multi-functional environment, so as to avoid the single function of the bus station, and it is convenient and practical. 


\section{Conclusion}

Through the design demonstration, it is found that the bus station changes the traditional single design concept, and the design details are integrated into people's lifestyle, which is popular for most people. In addition, making full use of energy conversion can not only protect the ecological environment, but also provide references for the design of bus station. Following conclusion are drawn by analyzing the present status quo and solution of the bus station design.

Nowadays, the bus station design cannot fully meet the sustainable development, few breakthrough and innovation are provided. Through analysis and prediction, it is found that the bus station and the public facilities are classified and integrated to achieve more rational resource optimization and utilization based on different needs of different cities in the design of the future bus station. The empirical study shows that the bus station can reduce the waste of resources and meet the humanization design with integration of social networking tools, information consulting and other daily facilities besides existing functionality. It is found that the integration of green accessory products and strengthen the details for specific groups in the design of bus station are popular in its application examples at abroad.

Therefore, the innovation and breakthrough of the bus station can not only be built on the city overall planning design concept and comprehensive system of government, but also need more people to strengthen the sense of ownership and protection and participate in the collection of opinions of the bus station design. The future bus station would adapt to international forms, and gradually form its own art products with geographical and cultural characteristics in the modernization, humanity, science and technology and information.

\section{Acknowledgments}

This research was carried out at the Sichuan Provincial Colleges and Universities Wood Industry and Furniture Engineering Key Laboratory, Sichuan Agricultural University.

\section{References}

1. A.B. Hu, The Research on Application of Experience Design for City Bus Station, MS thesis, Wuhan University Of Technology, Wuhan, 2012.
2. Z.F. Jiang, "Preliminary study on design of bus platform in modern city", Yihai, 2011(8), pp. 95-96.

3. L.X. Li, "The annotation and the resolution of the sustained development theories", Administration and Law, 2005(1), pp. 3-7.

4. X.W. Jun, The humanization design on bus station of public infrastructure, MS thesis, Nanchang University,Nanchang, 2010.

5. H. Zhao, Research on Humanized Design of the City Park, MS thesis, Taiyuan University of Technology, Taiyuan, 2011.

6. Y.N. Hou, and R.R Chen, "Influence of bus platform appearance on city image", TourismOverview, 2013(4), pp. 289-290.

7. F. Jiang, J.W.Xia and Y.Liu, "Optimization design and its application of bus stops based on traffic flow theory", Engineering Construction, 2014,46(1), pp. 17-20.

8. Y.W. Bian, Humanized Design of Bus Station in Modern Times, MS thesis, Nanchang University, Nanchang, 2014.

9. W. Zhong, The Research on Humanized Design of Street Sidewalk, MS thesis, Hunan University, Changsha, 2005.

10. H. Lv,HumanizationDesign of the City Public Space, MS thesis, TianjinUniversity, Tianjin, 2004.

11. Z. Wei, "To Create a Comfortable Seating Platform for Millions of Bus Group: The Humanized Design of Bus Shelter", Huazhong Architecture, 2012, (11), pp. 60-62.

12. M.Chen, et al, A kind of intelligent outdoor shed converting rain impact force into electrical energy: ChinaPatent ZL201520505526.6,2016-01-20.

13. J.H. Lyu, et al, A platform with temperature control and self washing function: ChinaPatent ZL201620024627.5,2016-06-08.

14. Yip, M, et al. "Exposure assessment of diesel bus emissions", International Journal of Environmental Research and Public Health, 2006(4), pp. 309-315.

15. Jaiswal, Sumeet, J. Bunker, and L. Ferreira. "Influence of Platform Walking on BRT Station Bus Dwell Time Estimation: Australian Analysis." Journal of Transportation Engineering, 2010(12), pp. 1173-1179.

16. Luigi T.De Luca, Propulsion physics (EDP Sciences, Les Ulis, 2009)

17. F. De Lillo, F. Cecconi, G. Lacorata, A. Vulpiani, EPL, 84 (2008) 